Article

\title{
Production of viable bovine embryos by intracytoplasmic sperm injection of oocytes harvested from slaughtered old cows
}

\author{
Mihai Cenariu ${ }^{1 *}$, Mihai Borzan ${ }^{1}$, Sorin Dan ${ }^{1}$, Remus Chiorean ${ }^{2}$ and Emoke Pall ${ }^{1}$ \\ 1 University of Agricultural Sciences and Veterinary Medicine Cluj-Napoca, Calea Mănăștur 3-5, Cluj- \\ Napoca, Romania; mihai.cenariu@usamvcluj.ro \\ 2 Stațiunea de Cercetare și Dezvoltare pentru Creșterea Bubalinelor Șercaia, Str. Câmpului nr.2, Șercaia, \\ Brașov, Romania; scdcb.sercaia@yahoo.com \\ * Correspondence: mihai.cenariu@usamvcluj.ro;
}

Received: 02.04.2021

Accepted: 16.04 .2021

Published: 21.04.2021

DOI: $10.52331 / c v j . v 26 i 1.8$

Copyright: (c) 2021 by the authors. Submitted for possible open access publication under the terms and conditions of the Creative Commons Attribution (CC BY) license (http://creativecommons.org/licenses /by/4.0/).

\begin{abstract}
Background: Intracytoplasmic sperm injection (ICSI) is currently used to increase fertilization success by avoiding several oocyte or sperm deficiencies that would normally prevent conception after in vivo fertilization or classical in vitro fertilization. This paper aimed at improving the in vitro fertilization protocol of bovine oocytes, harvested from old cows after slaughtering, using intracytoplasmic sperm injection; (2) Methods: Oocytes were harvested by puncture of follicles from ovaries obtained from slaughtered old cows, followed by aspiration. Out of the 127 cumulus-oocyte complexes that were harvested, $84(66.14 \%)$ were declared suitable for cultivation, after morphological evaluation. Following oocyte maturation for 22 hours, 77 cumulus-oocyte complexes were morphologically intact and could undergo the steps required for intracytoplasmic injection of spermatozoa. Frozen-thawed bull semen was used for ICSI and the 77 fertilized oocytes were kept for 24 hours in an atmosphere enriched with 5\% $\mathrm{CO}_{2}$.; (3) Results: Fertilized oocytes transformed into 46 zygotes (fertilization rate of $59.74 \%$ ), while after $168 \mathrm{~h}$ of cultivation 38 transferable compact morulae or early blastocysts were obtained; (4) Conclusions: Intracytoplasmic sperm injection can represent a viable alternative to classical IVF, when oocytes or sperm with lower fertility are used.
\end{abstract}

Keywords: bovine oocytes; ICSI; embryo; slaughtered cows.

\section{Introduction}

Intracytoplasmic sperm injection (ICSI) is currently used especially in assisted human reproduction as it was shown to be capable of increasing fertilization success by avoiding several oocyte or sperm deficiencies that would normally prevent conception after in vivo fertilization or classical in vitro fertilization (IVF) [1].

In cattle, the first successful ICSI was reported by Goto et al. in 1990 [2]. They used in vitro-matured (IVM) oocytes that were injected with immobilized bovine spermatozoa, and obtained embryos that developed in vitro up to the blastocyst stage and were further transferred to recipient cows that finally calved viable offspring.

Following this first successful attempt, several other researchers used this technique in order to enhance assisted reproductive performance of cattle with low quality gametes. An interesting study by Magata et al, 2019 [3], proved the beneficial effects of ICSI on the production of chromosomally normal embryos using oocytes harvested from aged cows. They showed that aging of females negatively influences the distribution of cortical granules during oocyte maturation, which could lead to abnormal fertilization, low developmental competence of oocytes, or/and increased aneuploidy. Such inconveniences could be surpassed by ICSI, thus allowing the prolongation of productive life and number of offspring obtained from valuable cows. 
Regarding male gametes, it is well known that classical IVF requires spermatozoa with normal morphology and intact function in order to obtain decent cleavage rates. Nevertheless, it was proven that ICSI can successfully represent a viable alternative when immature or suboptimal quality sperm was used in several species [4-7]. Therefore, ICSI could allow the use of lower quality semen, collected from younger bulls, as well as sexed semen for successful fertilization of oocytes [8].

Moreover, various reproductive disorders often lead to chronic complications, that permanently disrupt fertility and require culling of high yielding cows. In such females, not only the productive capacity is not fully capitalized, but also their potential to produce valuable offspring is hindered. Thus, the use of assisted reproductive technologies can aid in recuperating part of the losses, by harvesting the ovaries immediately after slaughtering and collecting the oocytes, followed by IVM and IVF/ICSI. Obviously, such expensive technologies are sustainable only in cows with high genetic merit, since their offspring will return all investments that were made. Therefore, the present study aimed at harvesting ovaries from slaughtered old cows, followed oocyte isolation and maturation as well as fertilization by ICSI using frozen/thawed bull semen and cultivation of resulted embryos until the morula or blastocyst stage.

\section{Materials and Methods}

The ovaries were harvested from 20 Holstein cows, aged 7-10 years, after slaughtering, and transported to the laboratory within 1 hour, in sterile saline, at $38^{\circ} \mathrm{C}$. Aspiration of the cumulus-oocyte complexes was performed using a $10 \mathrm{ml}$ syringe and a $22 \mathrm{G}$ needle (Figure 1 ).
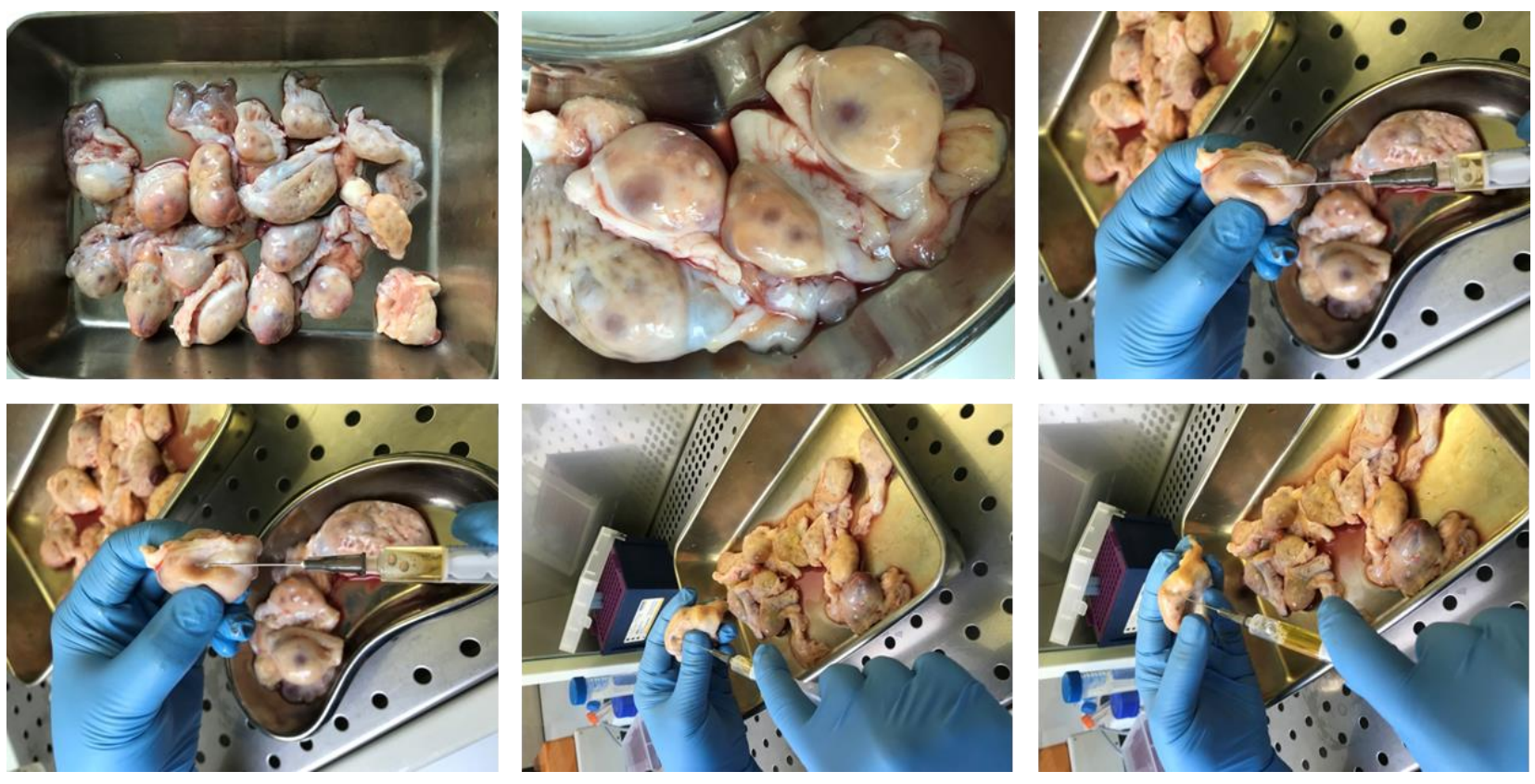

Figure 1. Needle aspiration of cumulus-oocyte complexes.

The follicular fluid was subsequently transferred to Petri dishes, which were examined using a stereomicroscope, in order to identify the cumulus-oocyte complexes and for morphological evaluation (to establish suitability for in vitro culture and fertilization) (Figure 2). Only oocytes that were surrounded by a large number of cumulus cells, arranged compactly around the zona pellucida, with a compact inner cell mass, without vacuolation and with a homogeneous appearance, were selected. Denuded oocytes, without a homogenous appearance, or with cytoplasmic vacuolation were discarded.

Oocyte maturation was performed in TCM199 (Sigma-Aldrich) supplemented with $4 \mathrm{mg} / \mathrm{ml}$ bovine serum albumin (BSA, Sigma-Aldrich), $0.1 \mathrm{IU} / \mathrm{ml} \mathrm{FSH}$ (Sigma-Aldrich) and $50 \mathrm{ng} / \mathrm{ml}$ epidermal growth factor (EGF, SigmaAldrich), at $38.5^{\circ} \mathrm{C}$, in an atmosphere with $5 \% \mathrm{CO} 2$ and $80-90 \%$ relative humidity, for 22 hours (Figure 3).

Oocyte denudation was done using a $0.1 \%$ solution of bovine testicular hyaluronidase (Sigma-Aldrich) for detachment and dispersion of cumulus cells, which were removed by careful pipetting (Figure 4). 
Semen straws were thawed at $37^{\circ} \mathrm{C}$ for 30 seconds and MTALP medium without bovine serum albumin (SigmaAldrich) supplemented with $5 \mathrm{mM}$ caffeine (Sigma-Aldrich) was added. Next, $10 \mu \mathrm{l}$ of the sperm suspension was mixed with $30 \mu \mathrm{l}$ of polyvinyl pyrrolidone solution PVP K90 (Sigma-Aldrich) at a concentration of 12\%.

A Narishige ONO-131 micromanipulator coupled to a Nikon Eclipse Ts2R microscope was used for intracytoplasmic sperm injection (Figure 5).
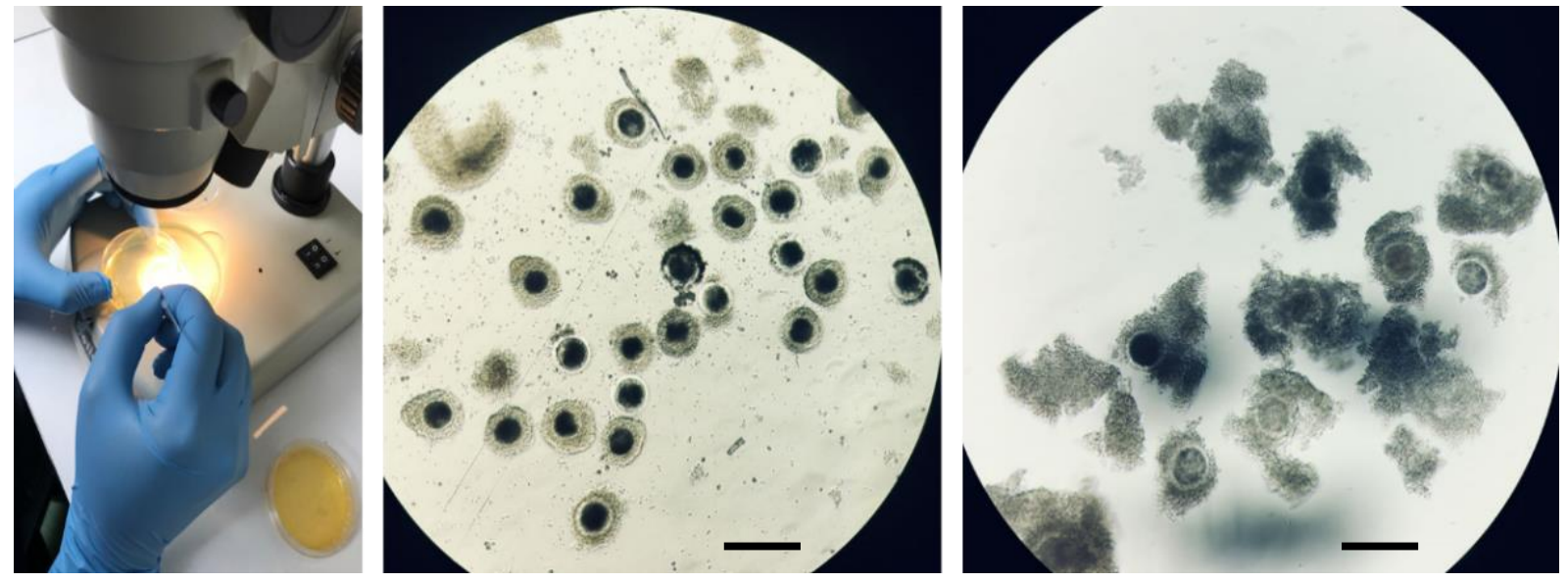

Figure 2. Morphological evaluation of cumulus-oocyte complexes (bar $=200 \mu \mathrm{m}$ ).
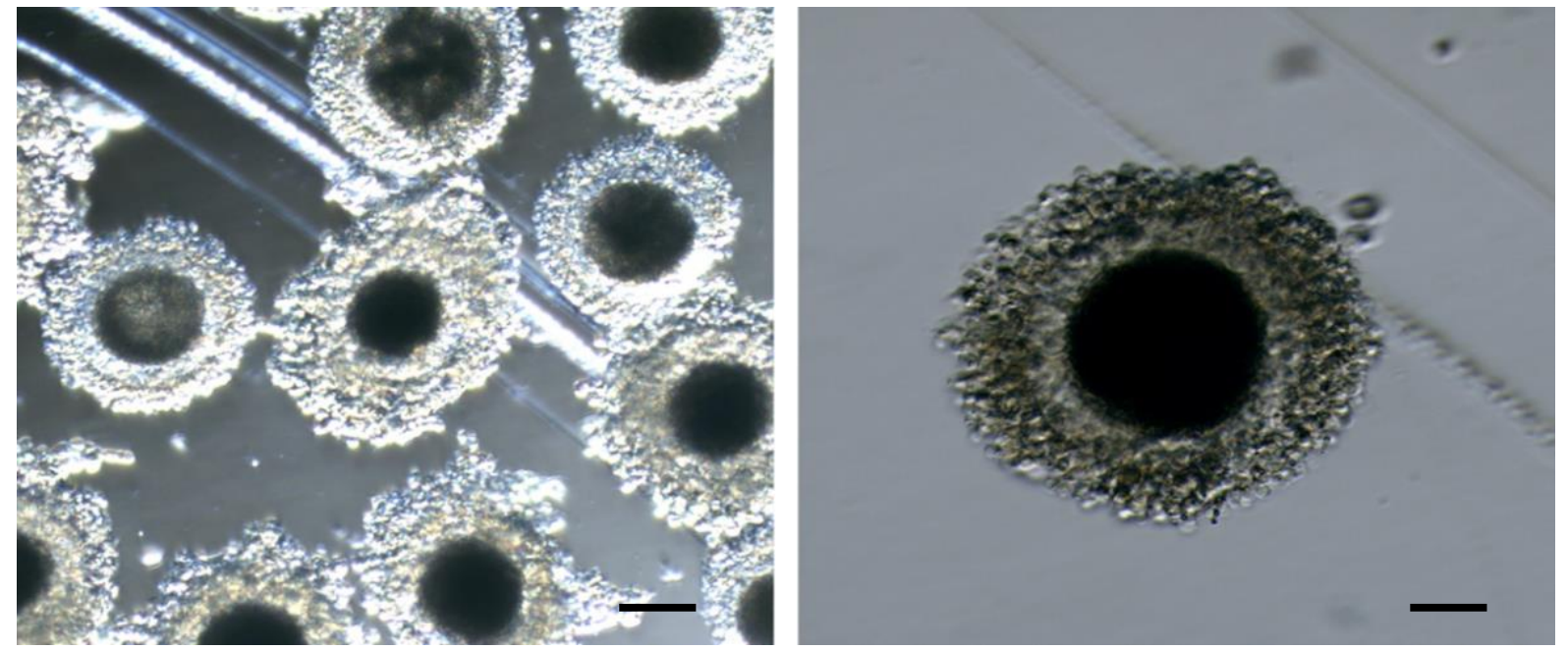

Figure 3. Bovine oocytes after maturation (bar=100 $\mu \mathrm{m}$ left, $50 \mu \mathrm{m}$ right).
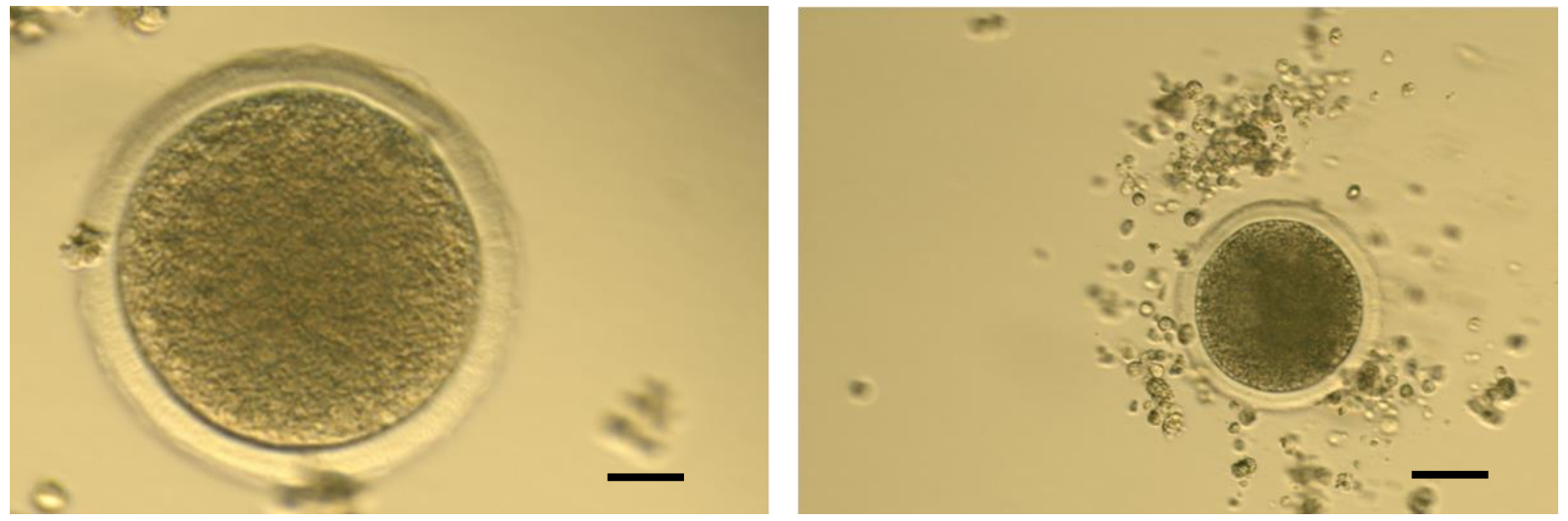

Figure 4. Bovine oocytes after denudation (bar=30 $\mathrm{m}$ left, $50 \mu \mathrm{m}$ right). 

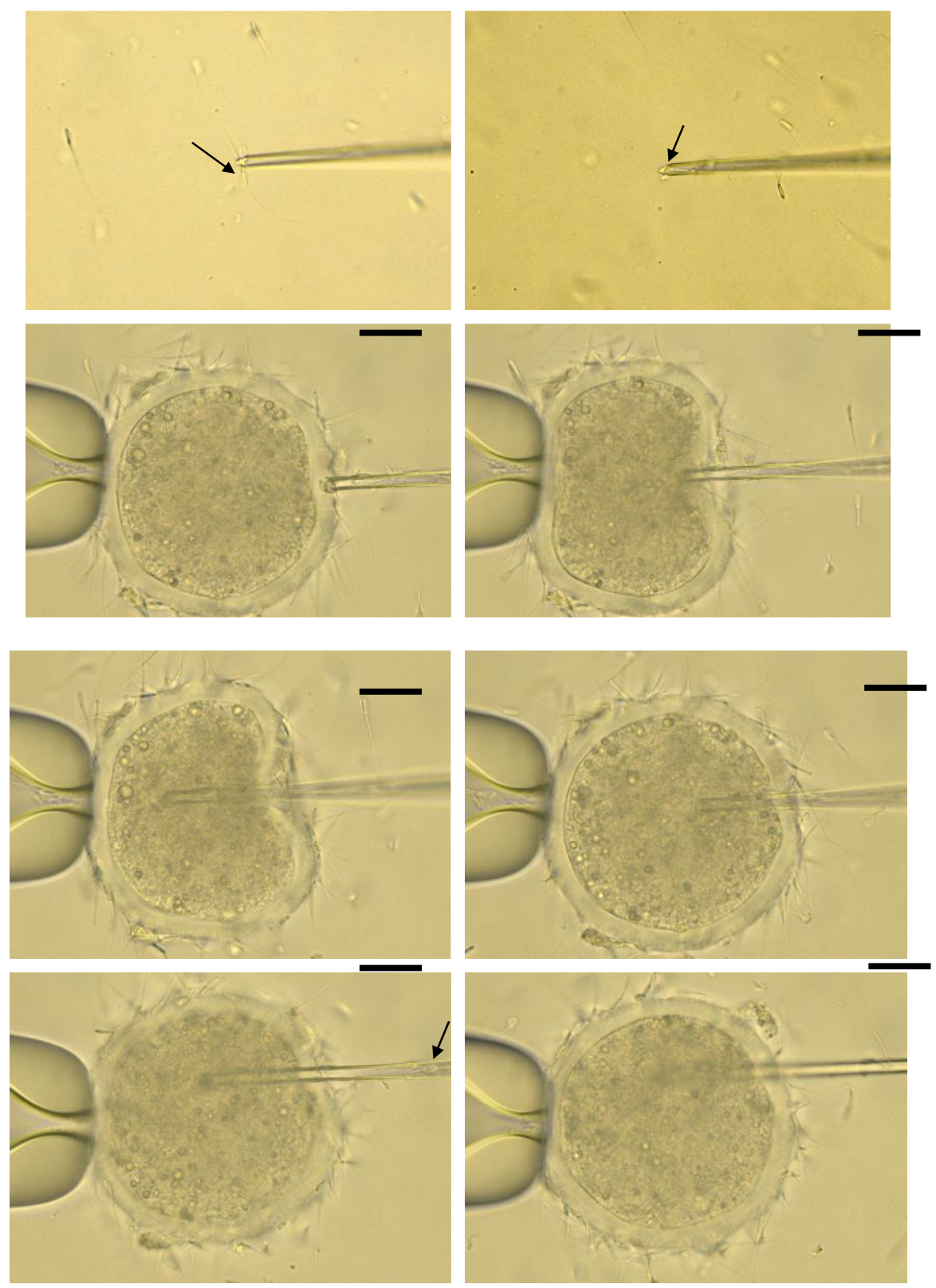

Figure 5. Intracytoplasmic sperm injection (arrow: spermatozoon, bar=30 $\mu \mathrm{m}$ ).

Cultivation of presumptive embryos was done in TCM199 supplemented with 5\% fetal bovine serum, at $38,5^{\circ} \mathrm{C}$, in $5 \% \mathrm{CO} 2$ and saturated relative humidity, for $24 \mathrm{~h}$. After $24 \mathrm{~h}$ developing embryos were moved in modified synthetic oviductal fluid, supplemented with $20 \mu \mathrm{l} / \mathrm{ml}$ essential amino acid solution, $10 \mu \mathrm{l} / \mathrm{ml}$ non-essential amino acid solution, $1 \mathrm{mM}$ glycine, $2 \mathrm{mM}$ taurine, $6 \mathrm{mg} / \mathrm{ml}$ bovine serum albumin and Insulin-Transferrin-Selenium supplement for 7 days in a trigas incubator: $5 \% \mathrm{CO} 2,5 \% \mathrm{O} 2$ and $90 \% \mathrm{~N} 2$.

\section{Results}

Out of the 40 ovaries, 127 cumulus-oocyte complexes were obtained (an average of 3.17 complexes/ovary) of which only 84 met the quality criteria required for cultivation (Figure 6).

Following the in vitro maturation process, 79 of the 84 cumulus-oocyte complexes showed morphological changes characteristic of maturation. 


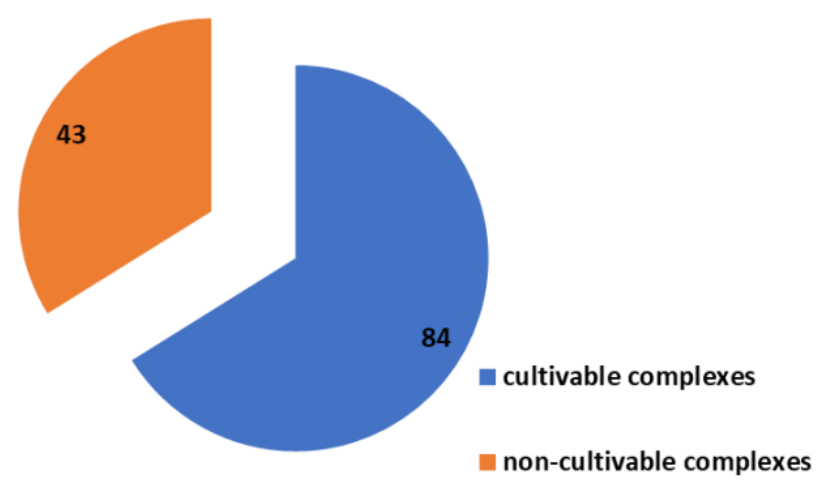

Figure 6. Morphologic evaluation of cumulus-oocyte complexes.

After denudation and careful microscopic examination, 2 oocytes were removed, as they showed morphological changes in the inner cell mass - 77 oocytes were subjected to the ICSI protocol.

After 24 hours culture, 46 of the 77 oocytes (59.74\%) were successfully fertilized (Figure 7).
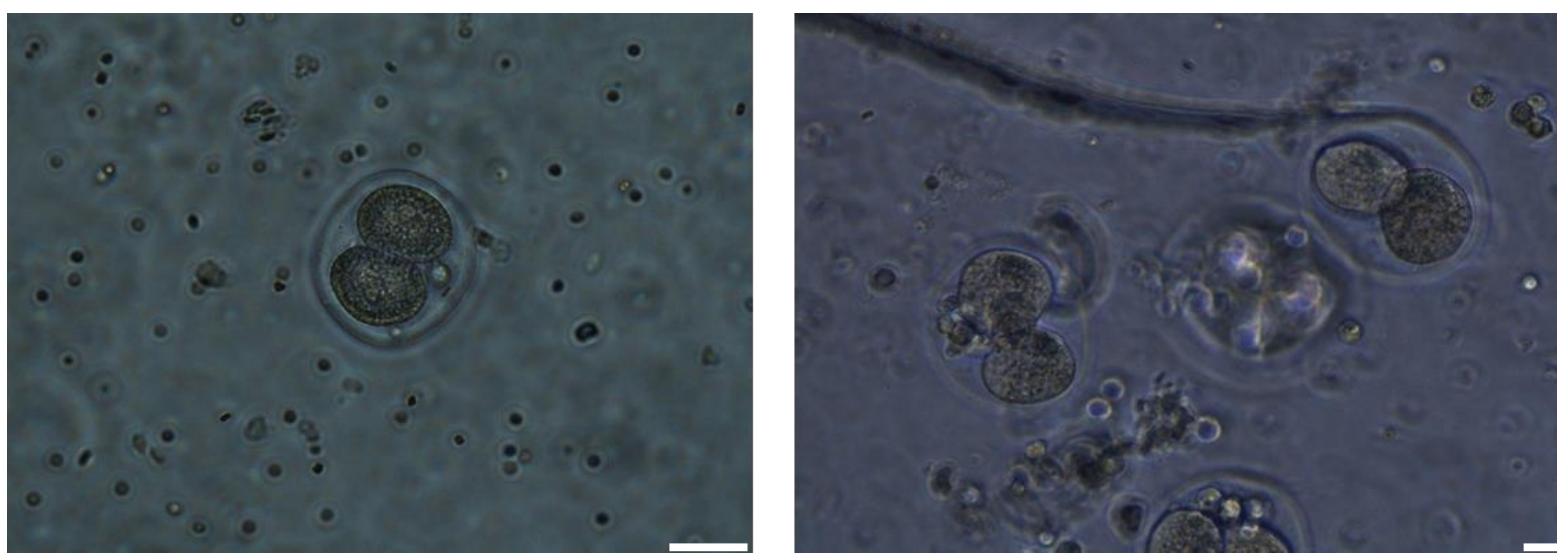

Figure 7. Viable embryos after $24 \mathrm{~h}$ of cultivation (bar=50 $\mu \mathrm{m})$.

After 168 hours, another 3 embryos degenerated, while the remaining 38 reached an age-appropriate developmental stage (compact morula or early blastocyst) and a normal morphology.
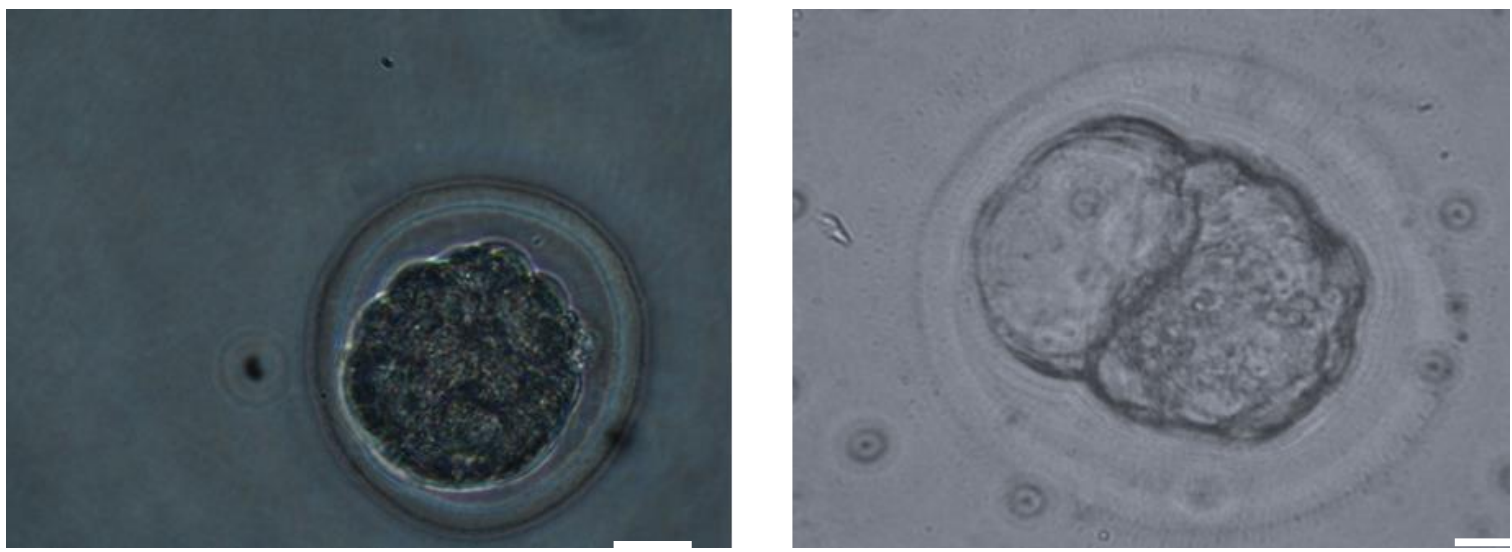

Figure 8. Viable embryos after $168 \mathrm{~h}$ of cultivation (left: compact morula, right: early blastocist, bar=30 $\mu \mathrm{m}$ ).

Therefore, the results of our research can be summarized as follows (Figure 9): 

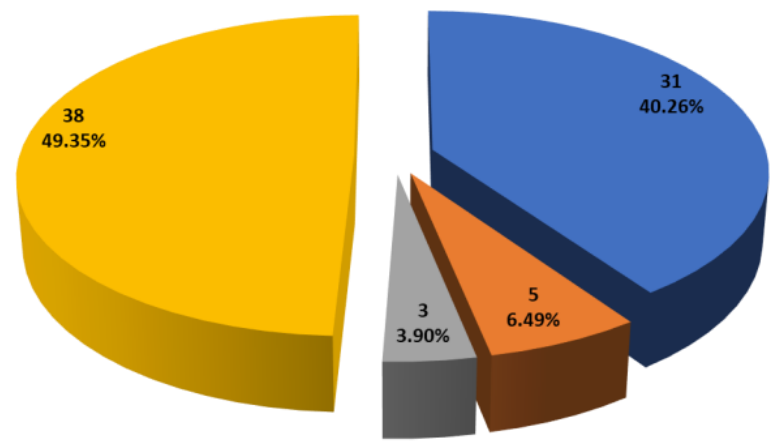

degenerated oocytes $24 \mathrm{~h}$ after ICSI

- degenerated embryos $72 \mathrm{~h}$ after ICSI

degenerated embryos 168 hours after ICSI

transferable embryos 7 days after ICSI

Figure 9. Results obtained after ICSI in bovine oocytes obtained from slaughtered old cows.

\section{Discussion}

The results obtained in this study bring more information on the development of the ICSI technique in cattle, as the literature is quite limited and often contradictory.

Thus, since the early 1980s there have been attempts to perform in vitro fertilization by ICSI technique in cattle, but the development of the zygote has stopped at the stage of pronucleus formation. A few years later, a group of Japanese researchers reported for the first time the success of fertilizing bovine oocytes with intracytoplasmic injected immobilized sperm and the birth of several normal and healthy calves [2]. In terms of embryo fertilization and production rates, the results obtained by ICSI were much poorer compared to conventional in vitro fertilization. This contrasted sharply with the experiences of those involved in human ICSI, in which sperm injection triggers seemingly normal fertilization and embryonic development. However, in cattle there have been many researchers who have believed that ICSI must necessarily be accompanied by an artificial method of activating oocytes to obtain a normal fertilization rate [9].

For this purpose, several methods of oocyte activation (using, for example, $7 \%$ ethanol, calcium ionophores, etc.) were tested in combination with ICSI $[10,11]$. Thus, bovine oocytes were activated by treatment with ionomycin (15 $\mathrm{mM}$ ) for 5 minutes and dimethylaminopurine (DMAP) for 4 hours. The same study was the first to report the production of blastocysts with normal karyotype following the injection of lyophilized bull sperm stored at $4^{\circ} \mathrm{C}$ until use.

Chung et al., In 2000 [12] tested an oocyte activation method that mimics the calcium oscillations observed in the normal fertilization process in cattle; oocytes were activated by three $5 \mathrm{~min}$ incubations with ionomycin at $30 \mathrm{~min}$ intervals. The study seems to confirm the findings that the ICSI technique itself is not sufficient to activate bovine oocytes, providing evidence in support of a partial activation called metaphase III (an abnormal stage in which chromosomes remain condensed after telophase II due to activation ooplasmic insufficiency). It has been speculated that such partial activation may occasionally provide sufficient cytoplasmic factors to initiate the formation of a female pronucleus, but insufficient for the processing of highly stable bovine sperm (containing type 1 protamine); it has also been speculated that partial activation may have occurred due to the fact that the sperm membrane remained intact or that, during the injection procedure, the ooplasmic membrane was not actually penetrated, but only surrounded the sperm, protecting its nucleus of the ooplasm-reducing medium.

Horiuchi et al., 2002 [13] reported that the use of immobilized bull sperm by breaking the tail and the use of a piezo-micromanipulator improved the ICSI result, with almost normal cleavage rates recorded after inoculation of oocytes that were activated by long ethanol exposure. for 5 minutes. Although Japanese researchers concluded that no activation procedure was required to perform fertilization, additional stimulation was required for blastocyst embryo development. The same researchers showed in another paper [14] that exposure of fertilized oocytes, which had two polar globules, to $7 \%$ ethanol for 5 minutes after ICSI, determined cleavage rates and blastocyst formation 
yields comparable to those obtained. in the case of conventional in vitro fertilization; they were also able to report the birth of five normal calves after the transfer of ten blastocysts to ten recipient cows.

In Korea, Hwang et al., 2000 [15] applied an electric shock to bovine oocytes before and after sperm injection; although the exact mechanism involved was not understood, electrical stimulation before and after ICSI has been shown to be effective in inducing oocyte activation and supporting embryonic development to the blastocyst stage.

Our study showed that the activation of fertilized oocytes is not mandatory, when obtaining blastocysts is not needed. If embryo culture stops on the 7th day after ICSI, most of them will be in the compact morula stage, which is perfectly suited for transfer or other manipulations (cryopreservation, sexing, etc.).

Sex sorting of bull semen by flow cytometry is currently a well-established technique, already applied in commercial practice in the US and Europe. In vitro fertilization requires a much smaller number of sperm than the artificial insemination technique, which is an advantage for this category of semen. However, there are studies that provide evidence of the viability and reduced motility of sorted sperm. It is known that proteins in the sperm membrane undergo changes that can affect its function and interactions with the oocyte after staining and sorting by flow cytometry [16]. It is also known that some bulls tolerate sperm sorting better than others [17]. Medvedev et al., 1997 [18] found some evidence that bull semen sorted by flow cytometry and cryopreserved was as effective as unsorted semen after intracytoplasmic injection. Skrzyszowska et al., 2000 [19] similarly reported that the yield of embryo formation does not differ between sorted and unsorted sperm following the application of the ICSI technique. Such results could suggest that sorting influences motility (which is not important for ICSI) rather than sperm fertilization capacity.

As mentioned above, ICSI in cattle could be very useful in the production of calves using expensive semen; there are bulls from which a single straw of frozen semen can cost up to 800EUR. Hundreds of oocytes could be injected from such a straw, and if the valuable semen was sexed as well, the efficiency would increase even more.

\section{Conclusions}

The oocyte collection technique from slaughtered cows' ovaries by puncture and aspiration, allowed the collection of a large number of cumulus-oocyte complexes (127 cumulus-oocyte complexes from 40 ovaries). Of the 127 cumulus-oocyte complexes collected, $84(66.14 \%)$ were declared cultivable, meeting the specific morphological assessment criteria. Following the maturation of the cumulus-oocyte complexes, a number of 77 morphologically suitable oocytes were obtained. After ICSI and culture for 24 hours, 46 zygotes were obtained, of which 38 continued their development for up to 7 days, reaching the morula or blastocyst stage. Thus, intracytoplasmic sperm injection can represent a viable alternative to classical IVF, when oocytes or sperm with lower fertility are used.

Author Contributions: Conceptualization and methodology, M.C. and E.P.; validation, M.B., R.C. and M.C.; formal analysis, M.C.; investigation, M.C., M.B., R.C., S.D. and E.P.; resources, S.D. and R.C.; writing-original draft preparation, M.C.; writing-review and editing, E.P. All authors have read and agreed to the published version of the manuscript".

Funding: Please add: This research received no external funding.

Conflicts of Interest: The authors declare no conflict of interest.

\section{References}

1. Fishel, S.; Aslam, I.; Lisi, F.; Rinaldi, L.; Timson, J.; Jacobson, M.; Gobetz, L.; Green, S.; Campbell, A.; Lisi, R. Should ICSI be the treatment of choice for all cases of in-vitro conception? Hum. Reprod. 2000, 15, 1278-1283. DOI: 10.1093/humrep/15.6.1278

2. Goto, K.; Kinoshita, A.; Takuma, Y.; Ogawa, K. Fertilisation of bovine oocytes by the injection of immobilised, killed spermatozoa. Vet. Rec. 1990, 127, 517-520.

3. Magata, F.; Tsuchiya, K.; Okubo, H.; Ideta, A. Application of intracytoplasmic sperm injection to the embryo production in aged cows. J. Vet. Med. Sci. 2019, 81(1), 84-90. DOI: 10.1292/jvms.18-0284

4. Wang, B.; Baldassarre, H.; Pierson, J.; Cote, F.; Rao, K.M.; Karatzas, C.N. The in vitro and in vivo development of goat embryos produced by intracytoplasmic sperm injection using tail-cut spermatozoa. Zygote 2003, 11, 219-227. DOI: $10.1017 / \mathrm{s} 0967199403002260$

5. Stein, P.; Schultz, R.M. ICSI in the mouse. Method Enzymol. 2010, 476, 251-262. DOI: 10.1016/S0076-6879(10)76014-6

6. Uehara, T.; Yanagimachi, R. Microsurgical injection of spermatozoa into hamster eggs with subsequent transformation of sperm nuclei into male pronuclei. Biol. Reprod. 1976, 15, 467-470. 
7. Lanzendorf, S.E.; Maloney, M.K.; Veeck, L.L.; Slusser, J.; Hodgen, G.D.; Rosenwaks, Z. A preclinical evaluation of pronuclear formation by microinjection of human spermatozoa into human oocytes. Fertil. Steril. 1988, 49, 835-842. DOI: 10.1016/s00150282(16)59893-8

8. Unnikrishnan, V.; Kastelic, J.; Thundathil, J. Intracytoplasmic Sperm Injection in Cattle. Genes 2021, 12, 198. DOI: 10.3390/ genes12020198

9. Oikawa, T.; Itahashi, T.; Numabe, T. Improved embryo development in Japanese black cattle by in vitro fertilization using ovum pick-up plus intracytoplasmic sperm injection with dithiothreitol. J. Reprod. Dev. 2016, 62(1), 11-16. DOI: 10.1262/jrd.2015-067

10. Keskintepe, L.; Pacholczyk, G.; Machnicka, A.; Norris, K.; Curuk, M.A.; Khan, I.; Brackett, B.G. Bovine blastocyst development from oocytes injected with freeze-dried spermatozoa. Biol. Reprod. 2002, 67, 409-415. DOI: 10.1095/biolreprod67.2.409

11. Meo, S.C.; Leal, C.L.V.; Yamazaki, W.; Garcia, J.M. Influence of simple and combined ionomycin, strontium and 6-DMAP treatments on activation and parthenogenetic development. Theriogenology 2002, 57, 706.

12. Chung, J.T.; Keefer, C.L.; Downey, B.R. Activation of bovine oocytes following intracytoplasmic sperm injection (ICSI). Theriogenology 2000 53, 1273-1284. DOI: 10.1016/S0093-691X(00)00271-5

13. Horiuchi, T.; Emuta, C.; Yamauchi, Y.; Oikawa, T.; Numabe, T.; Yanagimachi, R. Birth of normal calves after intracytoplasmic sperm injection of bovine oocytes: a methodological approach. Theriogenology 2002, 57, 1013-1024. DOI: 10.1016/s0093691x(01)00701-4

14. Horiuchi, T.; Emuta, C.; Oikawa, T.; Numabe, T. Comparison of bovine embryo yield following intracytoplasmic sperm injection and in vitro fertilization in individual bulls. Theriogenology 2000, 53, 393.

15. Hwang, S.; Lee, E.; Yoon, J.; Yoon, B. K.; Lee, J. H.; Choi, D. Effects of electric stimulation on bovine oocyte activation and embryo development in intracytoplasmic sperm injection procedure. J. Assist. Reprod. Genet. 2000, 17(6), 310-314. DOI: 10.1023/a:1009496726343

16. McNutt, T.L.; Johnson, L.A. Flow cytometric sorting of sperm: influence on fertilization and embryo/fetal development in the rabbit. Mol. Reprod. Dev. 1996, 43, 261-267. DOI: 10.1002/(SICI)1098-2795(199602)43:2<261::AID-MRD16>3.0.CO;2-6

17. Doyle, S.P.; Seidel, G.E, Jr; Schenk, J.L.; Herickhoff, L.A.; Cran, D.G.; Green, R.D. Artificial insemination of lactating Angus cows with sexed semen. J. Anim. Sci. 1999, 77(suppl.1), 99.

18. Medvedev, S.; Bossak, N.; Eckert, J.; Lucas-Hahn, A.; Niemann, H.; Johnson, L.A. Intra-cytoplasmic sperm injection (ICSI) with flowcytometrically sorted Y-chromosome bearingbovine sperm. Theriogenology 1997, 47, 270.

19. Skrzyszowska, M.; Shioya, Y.; Nagai, T.; Geshi, M.; Takenouchi, N. Development of cloned bovine embryos from nuclei of cumulus and muscle cell origin. Theriogenology 2000, 53, 244. 